vaccinia infective titres as are serum-containing lines. This, however, may be related to the decreased stability of small numbers of virus particles in a non-serum-containing medium. The important point in this study is that the final titre of vaccinia is similar in both cell lines. After four passages in serum-free cells the virus has not yet shown a greatly diminished infectivity for rabbits as has been reported in other serum-containing lines ${ }^{1}$.

Experiments are planned using other viruses in this same cell system. We are indebted to Dr. Wm. Cassel for provision of the vaccinia virus used in these studies.

Robert W. PUMpex

LAWrence J. AlFred

DAVID L. SACKETT

Department of Microbiology,

College of Medicine,

University of Illinois, Chicago.

${ }^{1}$ Cutchins, E., and Warren, J., Proc. Soc. Exp. Biol. Med., 97, 456 (1958).

${ }^{2}$ Hilleman, M. R et al., J. Amer. Med. Assoc., 163, 4 (1957). Salk, J. E., ibid., 158, 1239 (1955)

${ }^{3}$ McQuilkin, W. T., Evans, V. J., and Earle, W. R., J. Nat. Cancer Inst, 19, 885 (1957). Wavmouth, C., ibid., 22, 1003 (1959). Pumper, R. W., Science, 128, 363 (1958).

4 Younger, J. S., Proc. Soc. Exp. Biol. Med., 85, 202 (1954).

'Morgan, J. F., Morton, H. J., and Parker, R. C., Proc. Soc. Exp. Biol. Me.d., "73, 1 (1950).

Cassel, W. A. Virol., 3, 514 (1957)

'Sanford, K. K., Earle, W. R., and Likely, G. D., J. Nat. Cancer Inst., 9, 229 (1948)

s. Reed, I. 5., and Muench, H., Amer. J. Hyg., 27, 493 (1938).

- Pumper, R. W., Amer. J. Hyg., 69, 79 (1959).

\section{SCIENTIFIC METHOD}

\section{A Proposal to replace Belief by Method in the Pre-mensural Sciences}

Not only in his posthumous work, "Fundamental Theory"', but also in many of his earlier writings, Eddington was concerned to dofine the mathematical structure of what he called a 'measurable', and to distinguish a measurable from an observable, and an observable from the most primitive entity, which he regarded as resembling a geometrical point except that, instead of having position only (a far from primitive attribute), it has existence (or non-existence) only.

Eddington's proposed algebraic representations of these three concepts can be translated into the language of communication theory as follows : an entity has two characteristics, existence and nonexistence, and so can be represented by one binomial unit of information (bit). An observable is represented by two entities and has four characteristics, while a measurable is represented by two observables (or four entities) and has sixteen characteristics. This means that an entity has one way of not existing out of two possibilities, an observable three out of four, and a measurable fifteen out of sixteen.

We have devised ${ }^{2}$ an information model which represents the concept of a 'comparable', intermediate between an observable and a measurable. A comparable is represented by three bits of information, has eight characteristics, and accordingly it has seven ways of not existing within this universe of discourse.

The information structure (called the $\mathrm{O}$-structure, because it incorporates what is necessary for measur. ing or 'observing'-Eddington's $Z$ ) is a hierarchical structure. If the whole structure consists of $n$ bits there will be $\frac{1}{2} n$ in the ground-level, $\frac{1}{4} n$ in the next, and so on for the $L$ levels, where $L=\log _{2} n$, the number of bits in any level, $l$, being $n / 2 l$.

So long as a science is based upon measurables, its primitives must be measurable things, that is, atoms, but if a science is based upon comparables, this allows comparisons to be made between an observable entity, such as a semantic relationship with an observable, such as a physiological response. Luria's work ${ }^{3}$ along these lines makes meaning observable, but not measurable in the physical sense, and so, in principle, separates meaning from knowledge in just the same way as the cybernetic engineer separates information from meaning.

It is suggested that these separations now make it possible to formulate a scientific methodology of pre-mensural science, based upon similarity and comparable with the formal methodologies of dynamies and wave mechanics in the mensural sciences.

We represent the primitive entity, first by the binomial unit of information for ideal representation, and secondly as a two-way switch, in order that this ideal representation can be exemplified, at least in principle, by a cybernetic artefact. This double representation has been found capable of representing some characteristics of neural events, but owing to the great complexity both of these events and of experienced events (memories) it is not to be expected that detailed isomorphism can be established. However, it is easy to show how a connected system of switches can represent what is required, at least in a general way. If we start by assuming that all the connected switches have an equal probability of being open or closed, that is, a half-probability of existence, this will represent a system in which information or probability flow is everywhere maximal and uniform. If we now consider that some instability creates a linked succession of switches in the closed position, this will represent maximal existence and zero information. Such a linking inevitably produces a number of informationally isolated systems of zero existence and zero information; such systems represent events that are not being experienced. If we adopt Eddington's proposal that numbers between 0 and 1 represent degrees of statistical oxistence (phase) this allows us to suppose slight imperfections in our two types of system. The linking of chains of switches in the state of maximal existence may be imperfect (synaptic gaps), and as regards the isolated events that provide the possibility of recognition, these may be supposed to accumulate sufficient information by similarity to reach what might be called a 'semantic threshold' as a unit entity. It is such a unit entity that we envisage as the third element of a comparable, the other two being provided, in the case of perceptirn, by two micro-neural events or quanta that together constitute an observable.

\section{C. L. GreGcry}

Institute for the Study of Mental Images, Gally Hill,

Church Crookham, Hants.

'Eddington, A. S., "Fundamental Theory" (Camb. Univ, Press, 1946).

¿ Com. Inst. Study Mental Images. 1, Part 1, 1 (1957). Fregory, C. C. L. and Kohsen, A., "The O-Structure : an Introduction to Psychophysical Cosmology" (I.S.M.T., 1959). "Luria, A. R., and Vinogradova, O. S., Brit. J. Psychol., 50, 2, 89 